\section{INVESTIGACIÓN Y PATRIMONIO. CARTA ABIERTA A CONICYT Y DIBAM}

El trabajo de investigación y generación de nuevo conocimiento se alimenta y sustenta en una gigantesca red de soportes materiales y digitales. En esta línea son cruciales los laboratorios, museos, bibliotecas y centros de documentación, pero también los archivos y repositorios digitales.

Pese a los esfuerzos realizados en Chile en las últimas décadas, en nuestro trabajo de investigación constatamos cotidianamente brechas y problemas que, por una parte, dificultan dichos procesos investigativos y que, como consecuencia más general, por otra, limitan las potencialidades tecno-científicas del país, al mismo tiempo que amenazan gravemente nuestro patrimonio cultural, científico e intelectual.

En este sentido, nuestras experiencias nos sugieren algunas medidas que pueden ser adoptadas a corto y mediano plazo por los organismos competentes, CONICYT y DIBAM, y que proponemos a manera de abrir un debate sobre las tareas y áreas más urgentes de abordar:

1. Digitalización retrospectiva de todas las publicaciones científicas de Chile, accesible libremente para todo público.

2. Catalogación digital completa del acervo de nuestras bibliotecas y acceso mediante un buscador integrado.

3. Vinculación de los repositorios de las instituciones públicas.

4. Incorporación de fondos para libros y revistas en todos los proyectos de investigación y becas de posgrado, financiados por sistemas públicos.

5. Inclusión de financiamiento para la producción de al menos un fondo documental o archivo, digital o material, localizado en un museo, biblioteca o centro de documentación, para cada investigación y tesis de posgrado financiada por sistemas públicos, para las cuales sea pertinente dicha exigencia.

6. Consejos administrativos participativos para bibliotecas y centros de documentación.

7. Revisión y estandarización de las políticas de acceso a antiguas fichas clínicas y judiciales de hospitales públicos y juzgados. 
La evaluación e implementación de las medidas concretas aquí señaladas, concierne tanto a CONICYT como a DIBAM, organismos cuyo trabajo debería, a nuestro juicio, vincularse y articularse de modo más sistemático, en diversas áreas y niveles.

La formación de capital humano, el fortalecimiento de la base científica y tecnológica del país, así como su desarrollo científico e intelectual -todo lo cual está presente en los objetivos de CONICYT-, requiere, sin duda alguna, de mecanismos que le permitan incidir, al mismo tiempo que ser afectado por, el rescate, la conservación y la difusión del patrimonio nacional, contemplado en la misión de DIBAM.

Como investigadores/as preocupados/as e involucrados/as en el presente y el futuro de nuestro patrimonio intelectual, científico y cultural, creemos que tenemos la responsabilidad de contribuir a este empeño de conservación y promoción, por lo cual firmamos este documento e instamos a las instituciones públicas mencionadas, a evaluar las medidas esbozadas y comenzar un diálogo con la comunidad de investigadores/as nacionales a este respecto.

Santiago, 29 de julio de 2013.

Firman:

1. Silvana Vetö Honorato. Candidata a Doctora en Historia, Universidad de Chile / Becaria CONICYT, Profesora Titular Escuela de Psicología Universidad ARCIS.

2. Yuri Carvajal Bañados. Doctor en Salud Pública, Profesor Asistente Escuela de Salud Pública, Facultad de Medicina, Universidad de Chile.

3. María José Correa Gómez. Doctora en Historia, UCL / Posdoctoranda FONDECYT, Académica de Licenciatura en Historia, Universidad Andrés Bello.

4. Mariano Ruperthuz Honorato. Doctor (C) Psicología, Universidad de Chile. Académico Universidad del Desarrollo y Profesor Escuela de Psicología USACH. Miembro Comité Editorial de la Revista "Culturas Psi” (www.culturaspsi.org).

5. Carolina Bravo González.Doctoraen Historia, Universidad de Chile / Docente y Becaria CONICYT.

6. Vicente Painel Seguel. Licenciado y Profesor de Filosofía. Magíster en Historia, Universidad de Chile / Profesor de Aula en Liceos, Encargado Técnico Pedagógico de Escuela Rural. IX Región de La Araucanía.

7. Andrea Rojas Coll. Arquitecta Universidad Central de Chile / Magíster en Arquitectura PUC / Doctora@ en Arquitectura y Estudios Urbanos PUC.

8. César Leyton Robinson. Doctorando en Historia, Universidad de Chile / Curador del Museo Nacional de Odontología de la Facultad de Odontología de la Universidad de Chile y Académico de la misma Universidad.

9. Elisa Neumann García. Psicóloga, Magíster en Psicoanálisis / Docente Titular y Directora Escuela de Psicología, Universidad de Arte y Ciencias Sociales ARCIS.

10. Braulio Rojas Castro. Magíster en Filosofía mención Pensamiento Contemporáneo Universidad de Valparaíso, Doctor (C) en Filosofía Pontificia Universidad Católica de Valparaíso.

11. Carolina González Undurraga. Doctoranda en Historia, COLMEX. Académica Universidad de Chile.

12. Claudia Fedora Rojas Mira. Doctora en Estudios Americanos,especialidad Historia, IDEA, Facultad de Humanidades, Universidad de Santiago de Chile. 
13. José Luis García Fuentes. Doctorando en Salud Pública Universidad de Chile. Químico Farmacéutico. Coordinador curso Salud Pública, Química y Farmacia, Universidad de Chile / Becario MECESUP.

14. Esteban Radiszcz Sotomayor. Doctor en Psicopatología y Psicoanálisis, Universidad París VII. Académico Departamento de Psicología, Facultad de Ciencias Sociales, Universidad de Chile.

15. Sergio Witto. Editor. Profesor de la Escuela de Psicología, Facultad de Ciencias Sociales, Universidad Andrés Bello.

16. Cristián Idiáquez. Magíster en Psicología Universidad de Chile. Becario CONICYT Doctorado. Profesor universitario, miembro del equipo directivo Escuela de Psicología Grupal y Análisis Institucional "Enrique Pichon-Rivière”. Investigador colaborador Programa Clínica y Cultura, Universidad de Chile.

17. Aníbal Vivaceta de la Fuente. Profesor Auxiliar Departamento Salud Pública, Escuela de Medicina, Universidad de Valparaíso.

18. Marcelo Sánchez Delgado. Candidato a Doctor en Estudios Latinoamericanos, Universidad de Chile, Becario CONICYT. Grupo de Estudios en Historia de la Ciencia.

19. Andres Leiva G. Psicólogo. Doctor (C) en Procesos Sociales y Políticos en América Latina (PROSPAL) Universidad de Arte y Ciencias Sociales ARCIS. Editor revista Sujeto, Subjetividad y Cultura. Escuela de Psicología ARCIS.

20. María Josefina Cabrera. Candidata a Doctor en Historia PUC. Magíster en Historia.Académica Facultad de Educación PUC.

21. Diego Ortúzar R. Doctorante en Ciencias Sociales, Universidad de Buenos Aires/Escuela de Altos Estudios en Ciencias Sociales de París, Becario CONICET.

22. Karenlyn Mateluna Erazo. Profesora EGB, Periodista y Licenciada en Comunicación Social, Universidad de Playa Ancha de Ciencias de la Educación.

23. Catalina Valdés E. Doctoranda en Historia del Arte, Universidad de San Martín, Buenos Aires/ Escuela de Altos Estudios en Ciencias Sociales de París, Becaria CONICYT.

24. Marcelo Urra. Psicólogo. Docente Escuela de Psicología, Universidad ARCIS.

25. Sebastián Ligüeño E. Psicólogo, Doctorando en Psicología, Universidad de Chile. Docente Escuela de Psicología ARCIS.

26. Marcelo Valenzuela Cáceres. Bachiller en Humanidades. Profesor de Historia y Ciencias Sociales. Magíster Cen Historia, Universidad de Concepción.

27. Josefina de la Maza. Historiadora del Arte. Profesora del Departamento de Arte, Facultad de Filosofía y Humanidades, Universidad Alberto Hurtado. Doctora en Historia y Crítica de Arte, SUNY-Stony Brook, NY.

28. Maricela González. Asistente Social, estudiante de Doctorado en Historia, Universidad Católica de Chile.

29. Belén Gallo. Licenciada en Historia, Estudiante de Magíster en Estudios Latinoamericanos, Universidad de Chile.

30. Andrea del Campo Peirano. Licenciada en Historia, Pontificia Universidad Católica. Magíster en Psicología Comunitaria, Universidad de Chile.

31. Paulina Fernández Trabucco. Licenciada en Historia, Universidad Finis Terrae. Magíster en Sociología y Antropología, especialidad Políticas Culturales.

32. Helga Peralta Gamboa. Profesora de Castellano y Filosofía, ULS. Magíster en Filosofía $\mathrm{c} / \mathrm{m}$ Epistemología, Universidad de Chile. Becaria CONICYT, Doctorado en Filosofía c/m Estética y Teoría del Arte, Universidad de Chile. Docente Universidad Diego Portales y Universidad Central de Chile.

33. Iván Conejeros Espinoza. Bioquímico. Doctor en Ciencias Veterinarias, Universidad Austral de Chile. Investigador Posdoctoral FONDECYT. Universidad Austral de Chile.

34. Pía Uribe. Magíster en Psicología Clínica, Universidad de Chile. Docente Facultad de Psicología, Universidad Diego Portales. 